\title{
Behavioral Medicine in China
}

\author{
Joost Dekker • Bo Bai • Brian Oldenburg • \\ Chengxuan Qiu • Xuefeng Zhong
}

Published online: 5 June 2014

(C) International Society of Behavioral Medicine 2014

Behavioral medicine is a rapidly developing field of research in China. Research in this field addresses questions on basic bio-behavioral mechanisms; individual health and clinical interventions; and public health and health promotion. Scientific exchange between Chinese behavioral medicine researchers and colleagues elsewhere in the world is somewhat limited, mainly because of the language barrier, and some professional and cultural differences. Improved communication and collaboration will be of benefit to both Chinese researchers and researchers elsewhere.

With this goal in mind, a call for contributions to a special issue of the International Journal of Behavioral Medicine was published in September 2012. In response to the call, 43 manuscripts were submitted. We are pleased to present the

\section{J. Dekker}

Department of Psychiatry, VU University Medical Center, PO Box 7057, 1007 MB Amsterdam, The Netherlands

\section{J. Dekker $(\bowtie)$}

Department of Rehabilitation Medicine, VU University Medical Center, PO Box 7057, 1007 MB Amsterdam, The Netherlands

e-mail: j.dekker@vumc.nl

\section{B. Bai}

Institute of Neurobiology, Jining Medical University, Jining,

Shandong, People's Republic of China

\section{B. Oldenburg}

School of Population and Global Health, University of Melbourne, Melbourne, Australia

\section{Qiu}

Aging Research Center (ARC), Department of Neurobiology, Care Sciences and Society, Karolinska Institutet and Stockholm

University, Stockholm, Sweden

\section{Zhong}

Institute of Health Education, Anhui Center for Disease Control and Prevention, Hefei, China special issue on Behavioral Medicine in China, consisting of 20 interesting papers. Several manuscripts were not accepted: not addressing issues in behavioral medicine was by far the most frequent reason for not accepting these manuscripts. Most of these manuscripts addressed basic and clinical research questions in the field of mental health. Behavioral medicine and mental health are closely linked but distinct fields of research; apparently, these fields are less distinct in China than in other countries.

Three major commentaries introduce the field of behavioral medicine in China. Bai and Ji [1] describe the history of behavioral medicine in China and the development of the Chinese Society of Behavioral Medicine (CSBM). With regard to the future, they emphasize techniques commonly used in the prevention and treatment of somatic and psychological disorders in China. They focus on mechanisms underlying these interventions. Yang et al. [2] review common behavior modification techniques used in clinical settings in China. These include behavior therapy, cognitive therapy, cognitive behavioral therapy, health education, behavior management, behavioral relaxation training, stress management intervention, desensitization therapy, biofeedback therapy, and music therapy. These authors discuss possible biobehavioral mechanisms of these techniques. Ding et al. [3] describe the emergence of recent public health challenges in China, particularly those related to lifestyle-related non-communicable diseases. These authors also address the question about how behavioral medicine research and practice can be incorporated into the public health system in China in order to address these public health challenges.

Biobehavioral mechanisms are addressed in the papers by Liang et al. [4] and Welmer et al. [5]. These studies focus on the interactions between lipid profile and depression and between cardiovascular risk factors and functional dependence in cardiovascular disease among Chinese older people. 
Clinical issues are addressed in five papers. Wang, Lee, et al. [6] present a systematic review and meta-analysis on the effects of tai chi on psychological wellbeing in variety of chronic conditions (both somatic and mental disease) and healthy adults. Because cognitive behavioral therapy for insomnia has not been widely disseminated in China, Wang, Li, et al. [7] studied the outcome of medication in combination with a therapeutic approach drawn from traditional Chinese medicine-the low resistance thought induction sleepregulating technique. Yeung et al. [8] report on the association between coping self-efficacy and quality of life among Chinese cancer survivors. Luo et al. [9] and Nan et al. [10] report on the psychometric properties of Chinese version of the Illness Attitude Scales and the Subjective Happiness Scale, respectively.

Research on public health and health promotion issues covers smoking, issues related to weight (both underweight and overweight), reproductive health, and the impact of earthquakes. Ding et al. [11] conducted a systematic review of literature on correlates of smoking among Chinese girls and women. Ma et al. [12] aimed to estimate the prevalence of smoking and assess the accuracy of self-report smoking status in Chinese adolescents. Li, Borland, et al. [13] examined Chinese smokers' exposure to anti-smoking messages in a range of channels and the impact of exposure on subsequent quit attempts. Zhang et al. [14] assessed the mediation of smoking amount on the association of perception of smoking risks with successful spontaneous smoking cessation. Trends in the prevalence of underweight and overweight in Chinese youth from 2004 to 2009 are being reported by Seo and Niu [15]. Niu et al. [16] studied the discrepancy between the actual and perceived weight status and its relationship to dietary intake among Chinese youth. Li et al. [17] report on family correlates of overweight among Chinese children in urban areas. Two intervention studies focus on reproductive health and sexually transmitted diseases. Zhu et al. [18] implemented a community-based educational intervention targeting unmarried female migrant workers and studied the impact on reproductive health. Lau et al. [19] conducted a randomized controlled trial on the efficacy of a cognitive behavioral intervention in increasing prevalence of condom use with their male sex partner among Chinese monogamous female sexually transmitted disease patients. Psychosomatic disorders in children and adolescents exposed to the major earthquake in Wenchuan County in 2008 are the focus of the study by Sun et al. [20].

Together, these papers provide an in-depth perspective on behavioral medicine research in China. We trust that this special issue will contribute to scientific exchange between Chinese behavioral medicine researchers and colleagues elsewhere in the world.

Conflict of Interest Authors Dekker, Bai, Oldenburg, Qiu, and Zhong declare that they have no conflict of interest.

\section{References}

1. Bai B, Ji F. Behavioral medicine in China: history, current status, and future development. Int J Behav Med, this issue. doi:10.1007/ s12529-013-9331-0.

2. Yang Z, Su Z, Ji F, Zhu M, Bai B. Mechanisms of behavior modification in clinical behavioral medicine in China. Int J Behav Med 2014.

3. Ding D, Zhong X, Lau JT, Oldenburg B. Behavioral medicine and prevention of non-communicable diseases in China: current challenges and future directions. Int J Behav Med, this issue. doi:10. 1007/s12529-014-9393-7.

4. Liang Y, Yan Z, Cai C, Jiang H, Song A, Qiu C. Association between lipid profile and depressive symptoms among Chinese older people: mediation by cardiovascular diseases? Int J Behav Med, this issue. doi:10.1007/s12529-013-9358-2.

5. Welmer AK, Liang Y, Angleman S, Santoni G, Yan Z, Cai C et al. Vascular risk factor burden, atherosclerosis, and functional dependence in old age: a population-based study. Int J Behav Med, this issue. doi:10.1007/s12529-013-9352-8.

6. Wang F, Lee EK, Wu T, Benson H, Fricchione G, Wang W et al. The effects of Tai Chi on depression, anxiety, and psychological wellbeing: a systematic review and meta-analysis. Int J Behav Med, this issue. doi:10.1007/s12529-013-9351-9.

7. Wang W, Li G, Hong L, Liu Y, Zhao Y, Lin Y et al. Low resistance thought induction sleep-regulating technique (tip3-2) combined with medication for primary insomnia: a randomized controlled trial. Int $\mathrm{J}$ Behav Med, this issue. doi:10.1007/s12529-014-9415-5.

8. Yeung NC, Lu Q, Lin W. Specificity may count: not every aspect of coping self-efficacy is beneficial to quality of life among Chinese cancer survivors in china. Int J Behav Med, this issue. doi:10.1007/ s12529-014-9394-6.

9. Luo D, Zhang Y, Yu E, Tan Y, Tong Z, Zhou Y et al. The development and validation of a Chinese version of the illness attitude scales: an investigation of university students. Int J Behav Med, this issue. doi: 10.1007/s12529-014-9391-9.

10. Nan H, Ni MY, Lee PH, Tam WW, Lam TH, Leung GM et al. psychometric evaluation of the Chinese version of the subjective happiness scale: evidence from the Hong Kong FAMILY Cohort. Int J Behav Med, this issue. doi:10.1007/s12529-014-9389-3.

11. Ding D, Gebel K, Oldenburg BF, Wan X, Zhong X, Novotny TE. An early-stage epidemic: a systematic review of correlates of smoking among Chinese women. Int J Behav Med, this issue. doi:10.1007/ s12529-013-9367-1.

12. Ma J, Zhu J, Li N, He Y, Cai Y, Qiao J et al. Severe and Differential Underestimation of Self-reported Smoking Prevalence in Chinese Adolescents. Int J Behav Med, this issue. doi:10.1007/s12529-0139326-X.

13. Li L, Borland R, Yong HH, Fong GT, Jiang Y, Li Q et al. Reported exposures to anti-smoking messages and their impact on Chinese smoker's subsequent quit attempts. Int J Behav Med, this issue. doi: 10.1007/s12529-013-9349-3.

14. Zhang Y, Liu Y, Wang J, Jia C. Mediation of smoking consumption on the association of perception of smoking risks with successful spontaneous smoking cessation. Int J Behav Med, this issue. doi:10. 1007/s12529-013-9378-y.

15. Seo DC, Niu J. Trends in underweight and overweight/obesity prevalence in Chinese youth, 2004-2009. Int J Behav Med, this issue. doi: 10.1007/s12529-013-9332-z.

16. Niu J, Seo DC, Lohrmann DK. weight perception and dietary intake among Chinese youth, 2004-2009. Int J Behav Med, this issue. doi: 10.1007/s12529-013-9332-z.

17. Li B, Adab P, Cheng KK. Family and neighborhood correlates of overweight and obesogenic behaviors among Chinese children. Int $\mathrm{J}$ Behav Med, this issue. doi:10.1007/s12529-013-9333-y. 
18. Zhu C, Geng Q, Chen L, Yang H, Jiang W. Impact of an educational programme on reproductive health among young migrant female workers in Shenzhen, China: an intervention study. Int J Behav Med, this issue. doi:10.1007/s12529-014-9401-y.

19. Lau JT, Li J, Choi KC, Gu J. A Randomized controlled trial evaluating the efficacy of a theory-based intervention promoting condom use among
Chinese monogamous female sexually transmitted infection patients. Int J Behav Med, this issue. doi:10.1007/s12529-014-9388-4.

20. Sun XY, Fan HM, Bai B, Song HT, Tao FY, Song ZX et al. Psychosomatic conditions of the children and adolescents exposed to 5.12 Wenchuan earthquake. Int J Behav Med, this issue. doi:10. 1007/s12529-013-9377-z. 